\title{
Etnometodologia e comunicação: entrevista com Rod Watson
}

\section{Adriana Braga}

\section{Resumo}

Durante sua estada no Brasil em setembro de 2011, 0 Dr. Rod Watson concedeu esta entrevista exclusiva à E-Compós, na qual fala a respeito de sua carreira, da relação entre etnometodologia e comunicação e sobre questões teóricas e metodológicas de fenômenos da comunicação.
Adriana Braga I adrianabraga1@yahoo.com.br

Professora do Programa de Pós-Graduação em Comunicação Social da Pontifícia Universidade Católica do Rio de Janeiro (PUC-Rio). Pesquisadora do Conselho Nacional de Desenvolvimento Científico e Tecnológico (CNPq). Editora da E-Compós.
0 Professor Rod Watson nasceu em Halifax, Inglaterra, em 1943. É professor lecturer desde 1972 no Departamento de Sociologia da Universidade de Manchester. No transcurso de quase 30 anos, construiu reputação internacional como um dos mais destacados acadêmicos no campo da etnometodologia e da análise da conversação. Em 2009, recebeu o Prêmio Distinguished Research Publication, outorgado pela American Sociological Association.

Atualmente é pesquisador na Telecom ParisTech, na França. Em setembro de 2011, esteve no Brasil para ministrar um curso sobre Etnometodologia e Análise da Conversação, iniciativa conjunta dos Departamentos de Letras e Comunicação da PUCRJ e da UFRRJ. Durante esse período, concedeu esta entrevista exclusiva a Adriana Braga, editora da E-Compós.

Adriana Braga: Professor Watson, fale-nos, por favor, a respeito de como iniciou sua carreira e de algumas de suas influências.

Rod Watson: Fiz graduação e mestrado em sociologia na Universidade de Leicester, 
Inglaterra, que era, à época, a principal universidade da Europa nessa disciplina. $O$ chefe do Departamento de Sociologia era Ilya Neustadt, que foi, sem sombra de dúvida, o mais eminente professor de sociologia da GrãBretanha. Ele tornou a sociologia britânica muito mais cosmopolita, muito menos provinciana do que fora. Natural da Ucrânia, também estudou na Bélgica, de forma que fomos apresentados a uma série de sociologias da Europa Oriental e Ocidental e também a abordagens norteamericanas. Recebemos uma série de sociólogos visitantes do mundo todo, inclusive Gerhard Grohs da Alemanha e Reinhard Bendix dos EUA. Outro visitante, Peter I. Rose, do Smith College, EUA, ensinoume generosamente a fazer trabalho de campo em relações raciais, que fiz.

Entre meus professores estiveram Norbert Elias, um dos mais eminentes sociólogos do século XX, e o grupo que trabalhava com ele nesta perspectiva sobre o "processo civilizatório": o principal dentre estes foi Eric Dunning. Também aprendi com Anthony Giddens, agora Lorde Giddens, a respeito de Erving Goffman. Embora eu tenha levado muito tempo para perceber, os ensinamentos de Elias e Giddens foram influências decisivas para me refocar em questões relativas a comunicação, interação social, regras e conduta e na abordagem anti-cognitivista que Elias faz em sua brilhante crítica da distinção entre processos internos e externos.
Em nível de doutorado, primeiro na Universidade de Leeds e depois na Universidade de Warwick, trabalhei inicialmente com Robert Towler, fenomenologista da religião, e a seguir com John Heritage e Alan Dawe sobre a análise da comunicação como agência humana sob um ponto de vista etnometodológico e de análise da conversação. Minha tese foi sobre as comunicações telefônicas em tentativas de suicídio e dificuldades pessoais.

Primeiro trabalhei no Didsbury College, que então conferia diplomas da Universidade de Manchester, depois na Universidade de Lancaster por um ano que foi quase inteiramente nulo em termos intelectuais, $e$ depois na Universidade de Manchester.

Em Didsbury e Manchester, aprendi abordagens naturalistas, "sócio-antropológicas", da sociologia, inclusive etnometodologia de Garfinkel, análise da conversação Sacks, interacionismo simbólico / pragmatismo, as "Ethno Inquiries" de Rose, outras abordagens que se adicionaram aos veios Goffman / Elias de minha formação. Muitos sociólogos naturalistas eminentes estiveram em Manchester como professores visitantes. Os principais deles foram Howard S. Becker, Erving Goffman, Anselm Strauss, Joseph Bensman e também Harold Garfinkel, Harvey Sacks, Jim Schenkein, Gail Jefferson e outros. Todos eles foram muito importantes para o redirecionamento de meu olhar de sociólogo para a "virada linguística" considerada praxeologicamente. 
Como professor visitante no Buffalo State College da New York State University, trabalhei e publiquei com interacionistas simbólicos como Thomas S. Weinberg, Gale P. Largey e Herbert Auerbach sobre questões como a sociologia interacional dos sentidos e da comunicação, a comunicação de identidades sexuais e a análise de vídeos de interrogatórios policiais de suspeitos de assassinato. Este último projeto foi realizado com Georg Adoff.

\section{Adriana Braga: 0 campo dos Estudos da} Comunicação ainda é relativamente jovem e toma boa parte de suas teorias e métodos emprestada de outros campos disciplinares. $\mathrm{Na}$ interface entre Comunicação e Ciências Sociais, como o senhor avalia uma abordagem etnometodológica da comunicação?

Rod Watson: Esta é uma questão importante, e também é importante que os estudos da comunicação tenham se desenvolvido como campo. Mesmo agora, boa parte da sociologia ainda não deu a "virada linguistica": é como se as pessoas pudessem produzir ordem social sem serem capazes de comunicarem-se umas com as outras! Mesmo os sociólogos que assumiram essa virada, de certa forma só o fizeram da maneira mais abstrata e teórica, muitas vezes gerada por uma orientação de "Estudos Culturais" que nem sempre é tão útil. Por isto precisamos de estudos da comunicação como campo explicitamente definido. A comunicação é um campo, mais do que uma perspectiva disciplinar em si (para usar a distinção do filósofo Paul H. Hirst, em seu artigo incluído em um famoso livro editado por R. Archembault). Como tal, pode beneficiarse muito do ecletismo, de contribuições seletivas de várias disciplinas, como psicologia, sociologia, etc.

Assim, é uma pena que os estudos da comunicação às vezes sejam colonizados por outro campo, o dos estudos da mídia. Os estudos da mídia são um verdadeiro rolo compressor e vieram a dominar muitos aspectos dos estudos da comunicação. É claro que a mídia, inclusive a da internet, é muito importante, mas esta importância é com frequência altamente dramatizada pelos estudos da mídia, chegando às vezes a niveis apocalípticos. Contudo, ofato é que o fenômeno mais básico, mais ubíquo, é a comunicação interpessoal em contextos cotidianos - em interações domésticas familiares, na rua, no trabalho, em bares, ocasiões esportivas, etc.

Dada a natureza onipresente da troca discursiva na interação cotidiana, é irônico que muitos departamentos de Estudos da Comunicação subestimem ou até ignorem fenômenos tão importantes em favor $d a$ comunicação pela mídia. A etnometodologia e, particularmente, a análise da conversação / conversacional podem corrigir esse lamentável desequilíbrio. Essas abordagens podem proporcionar-nos análises ricamente detalhadas e específicas da comunicação em contexto 
sociais específicos da vida comum, obviando assim o "desdém pelo caso particular", como disse Wittgenstein (cujos últimos trabalhos influenciaram muitíssimo a etnometodologia britânica), de muitas disciplinas e campos. Essencialmente, a etnometodologia usa uma versão forte da etnografia, muitas vezes auxiliada por vídeo-dados sobre exemplos particulares de ações-em-contexto, e a análise da conversação usa áudio/vídeo-dados sobre uma série de trocas discursivas organizadas uma por uma (turn-in-turn), particularmente a da conversação ordinária, que tem primazia entre os sistemas. As principais orientações da análise da conversação foram a análise de categorias participação em contexto e, a que é a principal abordagem, a análise da sequência de afirmações em contexto. Estas contribuíram para uma abordagem radicalmente naturalista dos estudos da comunicação, e muitos analistas da conversação, como Anita M. Pomerantz, Steven Clayman e Paul L. Jalbert, trabalham em departamentos de Estudos da Comunicação.

\section{Adriana Braga: 0 senhor realizou recentes pesquisas em Cambridge para empresas de informação, tais como Microsoft e Xerox. Como a Etnometodologia analisa fenômenos de Interação Homem-Computador (HCI)?}

Rod Watson: Sim, trabalhei em diferentes graus com o laboratório de sistemas inteligentes da Rank Xerox do Reino Unido, em Cambridge, com o World Digital Research Centre, na
Universidade de Surrey, e com a Microsoft

Research do Reino Unido, em Cambridge.

Também sou Professor de Ciência Social na

Telecom ParisTech, em um departamento

focado no uso da tecnologia da informação e da comunicação tecnologia (TICs). Também orientei trabalhos de doutorado para o Laboratório de Computação da Universidade de Oxford, que resultaram em alguns artigos sobre HCI, inclusive um escrito para uma coleção editada por Adriana Braga ("CMC, Identidades e Género: teoria e método". Covilhã/Portugal: Editora da UBI, 2005) e um com Christian Greiffenhagen chamado "Visual Repairables in Visual Studies" (2009, vol. 8, n.1, pp. 65-90, Sage), além de artigos com Richard Harper (no prelo), que é Diretor de Microsoft Research Reino Unido e também é etnometodólogo! No entanto, meu envolvimento com essas empresas foi relativamente menor comparado com o de outros etnometodólogos e analistas da conversação.

Nos EUA, etnometodólogos como Lucy A. Suchman, Marilyn Whaler, Jack Whalen e Randy Trigg examinaram muitas características da inserção das TICs em ações e interações humanas em contextos específicos - com frequência (embora não sempre) contextos de trabalho. Seu trabalho contribuiu muito para o desenvolvimento de estudos sobre locais de trabalho e práticas de trabalho como campos distintos. 
A etnometodologia pode contribuir grandemente para a maneira como as pessoas aprendem a usar e entender as TICs no contexto de referência de uma determinada tarefa prática, p.ex., plotar a posição e o trajeto de trens em uma linha do metrô de Londres (ver o trabalho de C. Heath, P. Luffe J. Hindmarsh). Os etnometodólogos também têm analisado coisas como o trabalho de engenheiros de software ao projetar um software, as práticas de comunicação de pessoas em viseofones (ou videofones - ver Fornel) e em vídeoconferências: a gama de tópicos específicos é imensa, o que significa que boa parte da pesquisa etnometodológica é feita fora das universidades. Isto é bom, pelo menos no sentido de que resta muito preconceito contra a etnometodologia e a análise da conversação dentro das universidades, particularmente nos Departamentos de Sociologia mais antiquados, nos EUA, na Europa continental e no Reino Unido. O uso de dados em áudio e vídeo é uma especialidade da etnometodologia e da análise da conversação neste campo e tem obtido valiosos resultados. Assim, estes enfoques são as principais influências sobre os estudos de local de trabalho - muitas vezes as duas abordagens são combinadas neste campo, como Heath e outros mostraram.

Adriana Braga: Como adotar uma abordagem naturalista que focalize os fenômenos relacionados com a mídia?
Rod Watson: Vou centrar minha resposta na mídia TIC. Uma grande contribuição da etnometodologia e da análise da conversação foi a nova modelagem do usuário. Em particular, na França, Bernard Conein (Departamento de Sociologia da Universidade de Nice) e Christian Licoppe, e Marc Relieu (da Telecom ParisTech, Nice-Sofia-Antipolis) oferecem um curso conjunto de M.A. sobre a nova modelagem do usuário. $O$ usuário é concebido, não da maneira altamente abstrata, de cima para baixo, que os engenheiros de software costumam adorar (p.ex. em termos da sequência de escolhas de branching, em termos da teoria dos jogos ou em termos matemáticos), mas de um modo "do térreo para cima", com base em gravações reais de áudio-vídeo do uso da TICs por pessoas reais em contexto "natural" (comum). Nesses contextos, vemos que os usuários não são os "ingênuos culturais" nem os fantoches tão simplórios que Garfinkel diz também serem típicos das sociologias clássicas, em maior ou menor grau, mas, na verdade, atores sociais sutilmente competentes em relação ao uso do telefone celular.

Um exemplo-chave, de cuja pesquisa participei, é a forma como coconversationalistas remotamente distribuídos em telefones celulares atuam quando um interlocutor tem de dar orientações de localização a seu/sua coconversationalista. A solução do engenheiro para encontrar percursos ao celular tende a usar o algoritmo "navegação do carro por 
satélite", ou seja, a menor distância entre dois pontos. Contudo, esse algoritmo não é o escolhido por coconversationalistas que estão usando celulares em circunstâncias reais. O que usam, em vez disto, são imensas quantidades de conhecimento anterior - se quem dá a orientação sabe que quem a recebe conhece certas ruas e pontos de referência, o modo como quem dá a orientação imagina a correta posição de quem a recebe em relação a este estado de conhecimento, etc. Desta maneira, podemos conhecer o uso real, a vivência real, não apenas em relação aos celulares em si, mas de fazer coisas específicas, tarefas específicas com os celulares. Claro que isto reconfigura em grandíssima medida o modelo simplista habitual do usuário.

Como consequência - e isto nos leva de volta à última produção filosófica de Wittgenstein -, chegamos a um modelo anticognitivista do usuário como ator social (sobre esta questão geral, ver a edição especial de Theory, Culture and Society, vol. 25, no2, Março de 2008, intitulada "The Debate Over Cognitivism", editada por Jeff Coulter e por mim). Em vez de um modelo mentalista, do tipo processamento da informação, da maneira como o indivíduo entende, temos uma concepção de entendimento como prática cultural colaborativa em um contexto (de interação) social. Exemplo recente de uma análise escrita com base anticognitivista é uma das minhas, intitulada "Trust in Interpersonal Interaction and
Cloud Computing” ("Confiança na Interação Interpessoal e Computação em Nuvem") apresentada na conferência "Confiança e Computação em Nuvem", organizada conjuntamente pelo Departamento de Filosofia da Universidade de Cambridge e a Microsoft em abril deste ano. 


\section{Ethnomethodology and Communication:} an interview with Rod Watson

\section{Abstract:}

During his stay in Brazil in September 2011, Dr. Rod Watson gave this exclusive interview to E-Compós, in which he speaks of his career, on the relationship between ethnomethodology and communication and about theoretical and methodological issues of communication phenomena.

\section{Etnometodología y Comunicación:} una entrevista con Rod Watson

\section{Resumen:}

Durante su estancia en Brasil en septiembre de 2011, el Dr. Rod Watson dio esta entrevista exclusiva para E-Compós, en que el habla de su carrera, sobre la relación entre la etnometodología y la comunicación y cerca de los problemas teóricos y metodológicos de los fenómenos de la comunicación. 


\section{Expediente}

A revista E-Compós é a publicação científica em formato eletrônico da Associação Nacional dos Programas de Pós-Graduação em Comunicação (Compós). Lançada em 2004, tem como principal finalidade difundir a produção acadêmica de pesquisadores da área de Comunicação, inseridos em instituições do Brasil e do exterior.
E-COMPÓS I www.e-compos.org.br I E-ISSN 1808-2599

Revista da Associação Nacional dos Programas de Pós-Graduação em Comunicação.

Brasília, v.14, n.2, maio/ago. 2011

A identificação das edições, a partir de 2008 passa a ser volume anual com três números.

\section{CONSELHO EDITORIAL}

Afonso Albuquerque, Universidade Federal Fluminense, Brasil Alberto Carlos Augusto Klein, Universidade Estadual de Londrina, Brasil Alex Fernando Teixeira Primo, Universidade Federal do Rio Grande do Sul, Brasil Ana Carolina Damboriarena Escosteguy, Pontifícia Universidade Católica do Rio Grande do Sul, Brasil

Ana Gruszynski, Universidade Federal do Rio Grande do Sul, Brasil Ana Silvia Lopes Davi Médola, Universidade Estadual Paulista, Brasi André Luiz Martins Lemos, Universidade Federal da Bahia, Brasil Ângela Freire Prysthon, Universidade Federal de Pernambuco, Brasil Angela Cristina Salgueiro Marques, Faculdade Cásper Líbero (São Paulo), Brasil Antônio Fausto Neto, Universidade do Vale do Rio dos Sinos, Brasil Antonio Carlos Hohlfeldt, Pontifícia Universidade Católica do Rio Grande do Sul, Brasil Antonio Roberto Chiachiri Filho, Faculdade Cásper Libero, Brasil Arlindo Ribeiro Machado, Universidade de São Paulo, Brasil Arthur Autran Franco de Sá Neto, Universidade Federal de São Carlos, Brasil Benjamim Picado, Universidade Federal Fluminense, Brasil César Geraldo Guimarães, Universidade Federal de Minas Gerais, Brasil Cristiane Freitas Guttreind, Pontifícia Universidade Católica do Rio Grande do Sul, Brasi Denilson Lopes, Universidade Federal do Rio de Janeiro, Brasil Denize Correa Araujo, Universidade Tuiuti do Paraná, Brasil Edilson Cazeloto, Universidade Paulista , Brasil Eduardo Peñuela Cañizal, Universidade Paulista, Brasi Eduardo Vicente, Universidade de São Paulo, Brasi Eneus Trindade, Universidade de São Paulo, Brasil Erick Felinto de Oliveira, Universidade do Estado do Rio de Janeiro, Brasil Florence Dravet, Universidade Católica de Brasília, Brasil Francisco Eduardo Menezes Martins, Universidade Tuiuti do Paraná, Brasi Gelson Santana, Universidade Anhembi/Morumbi, Brasil Gilson Vieira Monteiro, Universidade Federal do Amazonas, Brasi Gislene da Silva, Universidade Federal de Santa Catarina, Brasil Guillermo Orozco Gómez, Universidad de Guadalajara Gustavo Daudt Fischer, Universidade do Vale do Rio dos Sinos, Brasil Hector Ospina, Universidad de Manizales, Colômbia Herom Vargas, Universidade Municipal de São Caetano do Sul, Brasil leda Tucherman, Universidade Federal do Rio de Janeiro, Brasil Inês Vitorino, Universidade Federal do Ceará, Brasi Janice Caiafa, Universidade Federal do Rio de Janeiro, Brasil Jay David Bolter, Georgia Institute of Technology Jeder Silveira Janotti Junior, Universidade Federal de Pernambuco, Brasil João Freire Filho, Universidade Federal do Rio de Janeiro, Brasi
John DH Downing, University of Texas at Austin, Estados Unidos José Afonso da Silva Junior, Universidade Federal de Pernambuco, Brasil José Carlos Rodrigues, Pontifícia Universidade Católica do Rio de Janeiro, Brasil José Luiz Aidar Prado, Pontifícia Universidade Católica de São Paulo, Brasil José Luiz Warren Jardim Gomes Braga, Universidade do Vale do Rio dos Sinos, Brasil Juremir Machado da Silva, Pontifícia Universidade Católica do Rio Grande do Sul, Brasil Laan Mendes Barros, Universidade Metodista de São Paulo, Brasil Lance Strate, Fordham University, USA, Estados Unidos Lorraine Leu, University of Bristol, Grã-Bretanha Lucia Leão, Pontifícia Universidade Católica de São Paulo, Brasil Luciana Panke, Universidade Federal do Paraná, Brasil Luiz Claudio Martino, Universidade de Brasilia, Brasil Malena Segura Contrera, Universidade Paulista, Brasil Márcio de Vasconcellos Serelle, Pontifícia Universidade Católica de Minas Gerais, Brasil Maria Aparecida Baccega, Universidade de São Paulo e Escola Superior de Propaganda e Marketing, Brasil

Maria das Graças Pinto Coelho, Universidade Federal do Rio Grande do Norte, Brasil Maria Immacolata Vassallo de Lopes, Universidade de São Paulo, Brasil Maria Luiza Martins de Mendonça, Universidade Federal de Goiás, Brasil Mauro de Souza Ventura, Universidade Estadual Paulista, Brasil Mauro Pereira Porto, Tulane University, Estados Unidos Nilda Aparecida Jacks, Universidade Federal do Rio Grande do Sul, Brasi Paulo Roberto Gibaldi Vaz, Universidade Federal do Rio de Janeiro, Brasi Potiguara Mendes Silveira Jr, Universidade Federal de Juiz de Fora, Brasil Renato Cordeiro Gomes, Pontifícia Universidade Católica do Rio de Janeiro, Brasil Robert K Logan, University of Toronto, Canadá

Ronaldo George Helal, Universidade do Estado do Rio de Janeiro, Brasil Rosana de Lima Soares, Universidade de São Paulo, Brasil Rose Melo Rocha, Escola Superior de Propaganda e Marketing, Brasil Rossana Reguillo, Instituto de Estudos Superiores do Ocidente, Mexico Rousiley Celi Moreira Maia, Universidade Federal de Minas Gerais, Brasil Sebastião Carlos de Morais Squirra, Universidade Metodista de São Paulo, Brasi Sebastião Guilherme Albano da Costa, Universidade Federal do Rio Grande do Norte, Brasil

Simone Maria Andrade Pereira de Sá, Universidade Federal Fluminense, Brasil Tiago Quiroga Fausto Neto, Universidade de Brasília, Brasi Suzete Venturelli, Universidade de Brasília, Brasil Valério Cruz Brittos, Universidade do Vale do Rio dos Sinos, Brasil Valerio Fuenzalida Fernández, Puc-Chile, Chile Veneza Mayora Ronsini, Universidade Federal de Santa Maria, Brasil Vera Regina Veiga França, Universidade Federal de Minas Gerais, Brasil

\section{COMISSÃO EDITORIAL}

Adriana Braga I Pontifícia Universidade Católica do Rio de Janeiro, Brasil Felipe Costa Trotta I Universidade Federal de Pernambuco, Brasil

CONSULTORES AD HOC

Bárbara Heller, Universidade Paulista, Brasil

Luciana Mielniczuk, Universidade Federal do Rio Grande do Sul, Brasil Micael Herschmann, Universidade Federal do Rio de Janeiro, Brasil EDIČ̃̃ DE TEXTO E RESUMOS I Susane Barros SECRETÁRIA EXECUTIVA I Juliana Depiné EDITORAÇ̃o ELETRÔNICA I Roka Estúdio TRADUÇÃO I Sabrina Gledhill, Sieni Campos, Robert Finnegan
COMPós I www.compos.org.br

Associação Nacional dos Programas de Pós-Graduação em Comunicação

Presidente

Julio Pinto

Pontifícia Universidade Católica de Minas Gerais, Brasi

juliopinto@pucminas.br

Vice-presidente

Itania Maria Mota Gomes

Universidade Federal da Bahia, Brasi

itania@ufba.br

Secretária-Gera

Inês Vitorino

Universidade Federal do Ceará, Brasil

inesvict@gmail.com 